\title{
A single dose of escitalopram blunts the neural response in the thalamus and caudate during monetary loss
}

\author{
Carolin A. Lewis, MSc; Karsten Mueller, PhD; Rachel G. Zsido, BA; Janis Reinelt, MD; \\ Ralf Regenthal, MD, PhD; Hadas Okon-Singer, PhD; Erika E. Forbes, PhD; \\ Arno Villringer, MD, PhD; Julia Sacher, MD, PhD
}

\begin{abstract}
Background: Selective serotonin reuptake inhibitors (SSRIs) show acute effects on the neural processes associated with negative affective bias in healthy people and people with depression. However, whether and how SSRIs also affect reward and punishment processing on a similarly rapid time scale remains unclear. Methods: We investigated the effects of an acute and clinically relevant dose $(20 \mathrm{mg})$ of the SSRI escitalopram on brain response during reward and punishment processing in 19 healthy participants. In a doubleblind, placebo-controlled study using functional MRI, participants performed a well-established monetary reward task at 3 time points: at baseline; after receiving placebo or escitalopram; and after receiving placebo or escitalopram following an 8-week washout period. Results: Acute escitalopram administration reduced blood-oxygen-level-dependent (BOLD) response during punishment feedback in the right thalamus (family-wise error corrected $[F W E] p=0.013$ at peak level) and the right caudate head $\left(p_{F W E}=0.011\right.$ at peak level) compared to placebo. We did not detect any significant BOLD changes during reward feedback. Limitations: We included only healthy participants, so interpretation of findings are limited to the healthy human brain and require future testing in patient populations. The paradigm we used was based on monetary stimuli, and results may not be generalizable to other forms of reward. Conclusion: Our findings extend theories of rapid SSRI action on the neural processing of rewarding and aversive stimuli and suggest a specific and acute effect of escitalopram in the punishment neurocircuitry.
\end{abstract}

\section{Introduction}

How our brain responds to reward and loss is a critical aspect of mood regulation. A blunted hedonic response to rewards or an enhanced sensitivity to loss can underlie negative bias in reward processing, which has been shown in patients with major depressive disorder (MDD) ${ }^{1}$ and anxiety disorders. ${ }^{2}$ Evidence from human and animal studies that manipulate serotonin levels provides robust support for the role of serotonin in modulating the neural circuit that underlies rewarding and aversive processing. ${ }^{3}$ This association between serotonin and neural processing of reward and loss may explain why selective serotonin reuptake inhibitors (SSRIs), which manipulate serotonergic activity, can help improve the processing capabilities of patients with MDD and anxiety disorders in the context of reward and/or punishment. However, the direction of association and specificity of this neural signal during positive and negative feedback remains to be clarified.
We know that SSRIs modulate serotonergic activity ${ }^{4}$ by blocking the serotonin transporter within a single dose of oral administration, ${ }^{5}$ and this leads to increased levels of extracellular serotonin. It has long been recognized that the acute administration of SSRIs reduces raphe neuron firing rates mediated by serotonin-1A autoreceptors, which become activated by increased extracellular serotonin levels, particularly in regions such as the dorsal raphe nucleus. ${ }^{6}$ This decrease in firing rates seems to be dose-dependent ${ }^{7}$ and region-specific; ${ }^{8}$ some studies have reported no change in serotonin levels at low SSRI doses. ${ }^{9}$ Although this negative feedback mechanism effectively controls serotonergic neuron firing rates and (partly) serotonin release, evidence from microdialysis studies ${ }^{10}$ indicates that the acute administration of SSRIs can increase extracellular serotonin levels in several projection regions, such as certain regions of the forebrain, with noteworthy variability likely based on region-specific differences in serotonin-1A receptors..$^{8,11,12}$

Correspondence to: J. Sacher, Max Planck Institute for Human Cognitive and Brain Sciences, P.O. Box 500355, 04303 Leipzig Germany; sacher@cbs.mpg.de

Submitted Jul. 6, 2020; Oct. 12, 2020; Revised Nov. 20, 2020; Accepted Dec. 12, 2020

DOI: $10.1503 /$ jpn.200121 
Two main models have been put forward for the role of serotonin in modulating the neural circuitry that underlies reward and punishment. The first centres on serotonin in an opponent role to dopamine, suggesting that while dopamine modulates reward processing, serotonin modulates aversive signals such as punishment and loss. ${ }^{13,14}$ Daw and colleagues ${ }^{13}$ proposed that phasic serotonin firing underlies prediction error for future punishment but not future reward. This theoretical framework ${ }^{15,16}$ is supported by empirical evidence from interventional studies that demonstrate potentiated responsiveness to loss or punishment but not to reward after a reduction in central serotonin levels. By using a novel fast-scan cyclic voltammetry procedure to identify serotonin signals in vivo while participants completed an investment task, Moran and colleagues ${ }^{17}$ provided further direct evidence that serotonin encodes loss-related prediction errors.

A second main theoretical approach suggests that serotonin has an overall inhibitory role in the reward system. ${ }^{18,19}$ Neuronal activity in the raphe nuclei in single-unit recordings correlates with both expected and received reward values (see Nakamura ${ }^{20}$ for a review). Raising extracellular serotonin levels reduces neural responses in the reward system. ${ }^{21,22}$ The inhibitory role of serotonin may arise from interactions with the dopamine system, given that parts of the mesolimbic dopamine system receive extensive serotonergic innervation. ${ }^{23,24}$ By using optogenetic stimulation of dorsal raphe nucleus serotonin input to the ventral tegmental area combined with the administration of an SSRI, Browne and colleagues ${ }^{25}$ found reduced reward-related response in rats. Thus, several lines of evidence suggest that enhanced serotonergic signalling may exert an overall inhibitory influence on the neural reward system.

By assessing the brain responses to reward and punishment stimuli following a serotonergic intervention, pharmacological functional MRI (fMRI) studies have begun to provide evidence for the role of serotonin in reward and loss processing in humans in vivo. Lowering serotonin levels via acute tryptophan depletion increases the blood-oxygenlevel-dependent (BOLD) response to errors during negative feedback in $\mathrm{fMRI}$ in the prefrontal cortex (PFC). ${ }^{26} \mathrm{~A}$ single dose of citalopram decreased the neural response to negative outcomes in the dorsomedial PFC and increased the neural response to negative outcomes in the left amygdala during a card gambling task. ${ }^{27}$ Using a loss/no-loss paradigm, Del-Ben and colleagues ${ }^{28}$ reported increased BOLD signals in the insula and decreased BOLD signals in the orbitofrontal cortex during loss avoidance after citalopram intake. The suggestion that serotonin mediates overall reward processing has also received empirical support. Marutani and colleagues ${ }^{29}$ found decreased BOLD signals in the insula, putamen and dorsolateral PFC in anticipation of rewards during a monetary incentive delay task after a single dose of the SSRI paroxetine.

Acute serotonergic modulation affects reward and punishment processing, but the net effect of an impairment or a facilitation is not entirely consistent across studies. Underlying reasons for this include the fact that not all of the studies investigated the influence of serotonergic manipulation on responses to reward and punishment in the same experiment. We lack a clear understanding of whether acutely enhanced serotonergic transmission specifically attenuates the brain response to loss or punishment in healthy humans, or if this attenuation centres on reward processing. Taking this next step is important not only for a better understanding of how serotonergic agents generate their antidepressant effect, but also to increase our knowledge of the role of serotonin for processing reward and punishment or loss in the human brain.

In this pharmacological fMRI study, we used a validated and simple monetary reward task in a double-blind, placebo-controlled, crossover design in healthy participants. We administered a single dose of $20 \mathrm{mg}$ escitalopram, a clinically relevant dose of one of the most widely prescribed SSRIs. ${ }^{30}$ The goal of the present study was to investigate whether an acute serotonergic challenge modulated BOLD responses in the main areas of the neural circuit implicated in monetary reward (ventral striatum and medial $\mathrm{PFC}^{31}$ ) and monetary loss (anterior insula, caudate, putamen, thalamus, anterior cingulate cortex ${ }^{32}$ ) and whether any changes in the neural response would be specific to the loss or punishment contrast, or extend to reward-specific feedback. Given the putative specificity of acute serotonergic depletion effects on the loss or punishment condition, we hypothesized that we would find decreased brain responsiveness to monetary loss or punishment but not to reward during acutely increased serotonergic transmission.

\section{Methods}

\section{Study design}

We used a double-blind, placebo-controlled, crossover design to investigate neural responses to a single oral dose of $20 \mathrm{mg}$ escitalopram (to reliably block $80 \%$ of serotonin transporters ${ }^{5}$ ) and placebo (mannitol and aerosol) in identical capsules provided by the pharmacy of Leipzig University Clinic in 19 healthy participants (9 women, 10 men), with a washout period between sessions of 8 weeks (see Figure 1A for an overview of the study design). On the first test day, participants underwent a baseline fMRI scan before initial drug administration. For the drug fMRI scans, we measured participants 3 to 4 hours after drug administration, during peak blood concentration of escitalopram. ${ }^{33}$ The study was conducted in accordance with the Declaration of Helsinki and approved by the institutional ethics committee of the University of Leipzig. All participants provided written informed consent before participating, and were compensated for participation at $€ 8$ per hour.

\section{Participants}

Twenty-four healthy participants (12 men, 12 women; age $25 \pm 2$ years [mean \pm standard deviation], range $21-29$ years; body mass index $23 \pm 2 \mathrm{~kg} / \mathrm{m}^{2}$ ) were recruited from the Max Planck Institute of Human Cognitive and Brain Sciences 


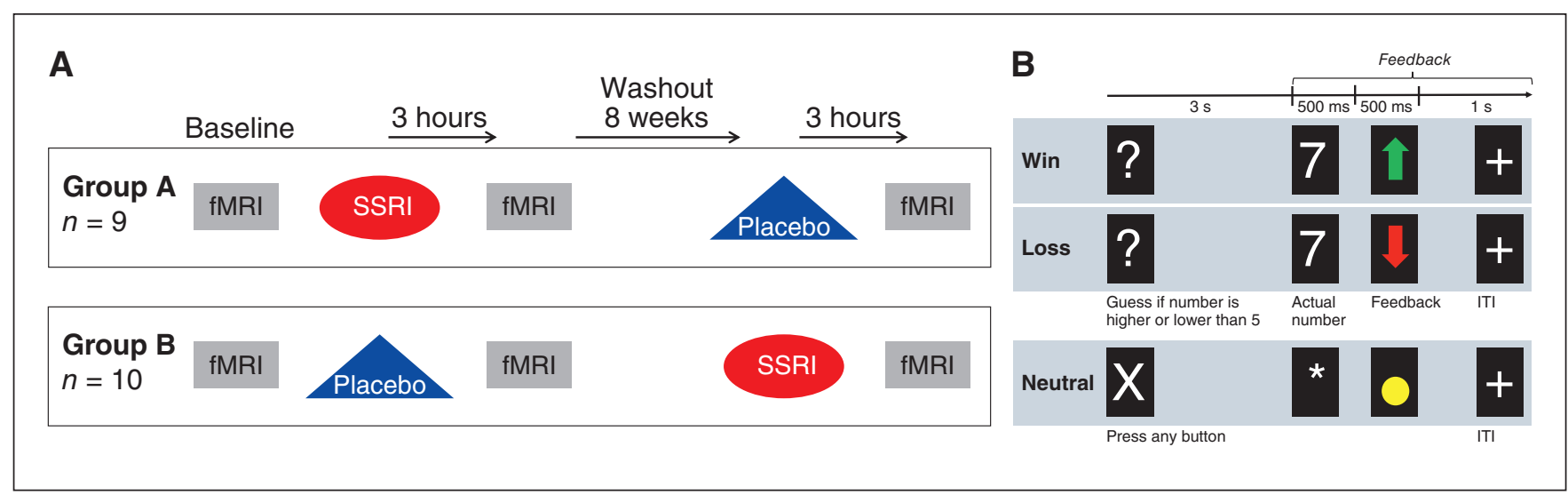

Fig. 1: (A) Study design. Nineteen participants underwent $3 \mathrm{fMRI}$ scanning sessions. After a baseline fMRI scan (grey square), participants received a single oral dose of the SSRI escitalopram (20 mg; red circle) or placebo (blue triangle) in a randomized design. We determined serum levels of escitalopram after 3 hours $\left(T_{\max }\right)$ and then conducted a second fMRI scan. After a washout period of 8 weeks, the protocol was repeated with the alternate study drug (escitalopram or placebo) to adhere to a double-blind intraindividual design. (B) Monetary reward task. The task consisted of 3 different block types (win, loss and neutral), with 45 trials in total. In win and loss trials, participants had $3 \mathrm{~s}$ to guess via button press whether the hidden number (between 1 and 9) on a visually presented card would be higher or lower than 5 . Then, the actual number was displayed for $500 \mathrm{~ms}$ and participants received outcome feedback for $500 \mathrm{~ms}$ (a green up-arrow for win outcomes or a red down-arrow for loss outcomes). Each trial ended with a crosshair symbol presented in the middle of the screen for $1 \mathrm{~s}$ (intertrial interval). In neutral trials, participants were asked to press a button when an $\mathrm{X}$ was displayed ( $3 \mathrm{~s}$ ), followed by an asterisk ( $500 \mathrm{~ms}$ ) and a yellow circle for neutral outcomes $(500 \mathrm{~ms}) . \mathrm{fMRI}=$ functional $\mathrm{MRI} ; \mathrm{ITI}=$ intertrial interval; SSRI = selective serotonin reuptake inhibitor; $\mathrm{T}_{\max }=$ time to peak concentration.

and the University of Leipzig. Exclusion criteria were pregnancy (urine pregnancy test at screening and before each test session); current or previous major psychiatric disorders (assessed using the Semi-structured Clinical Interview for Diagnostic and Statistical Manual of Mental Disorders, 4th edition, Axis I disorders, ${ }^{34}$ by a trained psychiatrist); the use of medications that might interfere with the study medication (e.g., antidepressants, antipsychotics, sedatives); chronic or acute physical illness (abnormal physical examination, electrocardiogram, or hematological and chemical blood analyses); tobacco smoking; lifetime illicit drug use more than 10 times (except for $\Delta^{9}$-tetrahydrocannabinol); illicit drug use within the last 2 months; and illicit drug use during the study (determined by urine drug tests). Subclinical depressive, hypomanic or anxiety symptoms were assessed using the 17-item Hamilton Depression Rating Scale ${ }^{35}$ by a trained psychiatrist, the self-report version of the Structured Clinical Interview for Mood Spectrum (MOODS-SR) ${ }^{36}$ and the State-Trait Anxiety Inventory. ${ }^{37}$ All participants were naive to antidepressants and free of medications except for the contraceptive pill.

Of the 24 enrolled participants, 1 was excluded because of a preanalytical error in acquisition of the serum sample, and 4 were excluded because of missing or corrupted log files from the presentation software. We analyzed the data from 19 right-handed participants with a normal weight (10 men, 9 women; age $24 \pm 2$ years, range $21-29$ years; body mass index $23 \pm 2 \mathrm{~kg} / \mathrm{m}^{2}$ ). Serum values of escitalopram $(\mathrm{ng} / \mathrm{mL})$ were acquired at peak concentration $\left(\mathrm{T}_{\max }\right)^{33}$ in blood and determined using liquid chromatography with UV detection. The quantification limit for escitalopram was $1 \mathrm{ng} / \mathrm{mL}$.

\section{fMRI paradigm}

We used a well-established monetary reward task, which elicits reliable activation in the neural reward circuitry and was designed to index the neural response to feedback about wins (rewards) and losses (punishment). ${ }^{38,39}$ The task consisted of 3 block types (win, loss and neutral), with 45 trials in total. Participants were told that their performance would determine a post-scan monetary reward: $€ 1$ for each win and $€ 0.50$ deducted for each loss. The blocks were presented in fixed pseudorandomized order with identical predetermined outcomes across participants (Figure 1B). Participants were unaware of the fixed outcome probabilities $(80 / 20$; i.e., $80 \%$ win outcomes and $20 \%$ loss outcomes in a win block and vice versa in a loss block).

In win and loss trials, participants had $3 \mathrm{~s}$ to guess via button press whether a hidden number (between 1 and 9) on a visually presented card would be higher or lower than 5. Then, the actual number was displayed for $500 \mathrm{~ms}$ and participants received outcome feedback for $500 \mathrm{~ms}$. For win outcomes, feedback was a green up-arrow, and for loss outcomes, feedback was a red down-arrow. A trial ended with a crosshair symbol presented in the middle of the screen for $1 \mathrm{~s}$ (intertrial interval). In neutral trials, participants were asked to press a button when an " $X$ " was displayed ( $3 \mathrm{~s}$ ), followed by an asterisk ( $500 \mathrm{~ms}$ ) and a yellow circle for neutral outcomes $(500 \mathrm{~ms})$. The total length of a trial was $5 \mathrm{~s}$. Before each block started, participants viewed instructions for the win, loss or neutral condition for $3 \mathrm{~s}$; this was not analyzed further.

Based on our hypotheses, the contrasts of interest derived from the task were win-neutral and loss-neutral. 
The feedback phase was defined as the intervals that included the number presentation and the feedback arrow. Similarly, the feedback phase in the control condition was defined as the interval between the display of an asterisk and a yellow circle. If the durations of the events of interest are the same and are less than $2 \mathrm{~s}$, it is common practice to model those events with a length of $2 \mathrm{~s}$ in further analyses. ${ }^{40}$

\section{Neuroimaging data collection and preprocessing}

We acquired MRI data using a 3 T Verio 3 scanner (Siemens), equipped with a 32-channel head coil, at the Day Clinic for Cognitive Neurology, University of Leipzig Medical Center. We acquired functional $T_{2}{ }^{*}$-weighted images using a gradient echo echo-planar imaging sequence with the following parameters: repetition time $2000 \mathrm{~ms}$; echo time $30 \mathrm{~ms}$; flip angle $90^{\circ}$; field of view $256 \times 256$; 30 axial slices; slice acquisition matrix $64 \times 64$; slice thickness $4 \mathrm{~mm}$; voxel resolution $3 \times 3 \times$ $4 \mathrm{~mm}^{3}$. Functional images were coregistered to $T_{1}$-weighted images, obtained using a magnetization-prepared rapid gradient echo sequence with the following parameters: repetition time $2300 \mathrm{~ms}$; inversion time $90 \mathrm{~ms}$; echo time $2.98 \mathrm{~ms}$; flip angle $9^{\circ}$; voxels $1 \times 1 \times 1 \mathrm{~mm}^{3}$. We scanned every participant 3 times: at baseline (without medication), with placebo and with escitalopram (randomized treatment order; for study design overview, see Figure 1A).

We preprocessed and analyzed data sets using SPM12 (Wellcome Trust Centre for Neuroimaging) and Matlab (The MathWorks Inc.). Preprocessing comprised head motion correction using realignment, including unwarping to correct for echo-planar imaging distortions using voxel displacement maps from the Fieldmap toolbox in SPM. We also checked if the degree of motion varied between scanning sessions by computing frame-wise displacement (FD). ${ }^{41}$ As an input, we used the translational and rotational motion parameters obtained by motion correction in SPM. For the full series of 150 functional images, motion between volumes was characterized using 149 FD values for each participant and both sessions. Finally, all FD time courses were characterized by the mean FD, the maximum FD, the maximum FD after eliminating the largest $5 \%$ of the FD values and the number of FD values that exceeded $0.5 \mathrm{~mm}$. We then analyzed mean and maximum FD to detect systematic motion differences between the SSRI and placebo scans across participants using paired-sample $t$ tests (2-tailed). For all participants and both sessions, the mean FD was less than $0.5 \mathrm{~mm}$, and we found no significant differences in FD parameters between the SSRI and placebo sessions. No participants were excluded for excessive motion. Further preprocessing steps included slicetime correction, coregistration with the mean anatomic image, and normalization to the Montreal Neurological Institute (MNI) space based on the unified segmentation approach. ${ }^{42}$ We resampled functional images in the MNI space with a resolution of $2 \times 2 \times 2 \mathrm{~mm}^{3}$. Finally, we performed spatial filtering using a Gaussian kernel with $8 \mathrm{~mm}$ full width at half maximum.

\section{Neuroimaging data analysis}

We analyzed preprocessed functional images using an eventrelated design in a general linear model to analyze the hemodynamic response to reward and punishment feedback at peak escitalopram plasma concentration. For each participant and scan, we performed parameter estimation and generated contrast images for the contrasts of interest (win-neutral and loss-neutral, trial by trial). We then included these first-level contrast images in a second-level analysis using a paired design. Thereafter, we computed statistical analyses using each contrast of interest (win-neutral and loss-neutral) to investigate potential differences between SSRI and placebo administration. After using an initial voxel threshold of $p<$ 0.001, we obtained significant results with family-wise error (FWE) correction at peak level at $p<0.05$, given previous work $^{43}$ showing that the cluster inference is prone to producing false-positive results but the voxel inference shows FWE rates in the expected order of magnitude. We performed all analyses at the whole-brain level.

\section{Results}

Sample characteristics, mood questionnaires and drug levels

Table 1 summarizes the demographic characteristics of the 19 healthy participants who completed the study protocol. We found no significant changes in depression and mood scores after the single dose of escitalopram compared to placebo.

Mean ( \pm standard deviation) plasma levels of escitalopram were in the expected range $(23 \pm 6 \mathrm{ng} / \mathrm{mL})$ when fMRI scans took place (3-4 hours after drug administration).

\section{Neuroimaging results}

Investigating the loss-neutral contrast, we found a diminished BOLD response after a single $20 \mathrm{mg}$ dose of escitalopram in the right posterior thalamus (cluster-size $k=$ 197 voxels; $\mathrm{T}_{\max }=7.7 ; p_{\mathrm{FWE}}=0.013$ at peak level; $p_{\mathrm{FWE}}=0.086$ at cluster level; MNI coordinates $x, y, z=10,-26,4)$ and the right caudate head $\left(k=424\right.$ voxels; $\mathrm{T}_{\max }=7.9 ; p_{\mathrm{FWE}}=0.011$ at peak level; $p_{\mathrm{FWE}}=0.005$ at cluster level; MNI coordinates

Table 1. Sample demographics and depression and mood scores

\begin{tabular}{lcc}
\hline Characteristic & Finding $^{*}$ & $t$ value; $p$ value \\
\hline Demographics & $24 \pm 2$ & - \\
Age, yr & $23 \pm 2$ & - \\
Body mass index, $\mathrm{kg} / \mathrm{m}^{2}$ & $33 \pm 8$ & - \\
State-Trait Anxiety & & \\
Inventory, trait anxiety & & $t_{1,17}=0, p=1$ \\
Rating scales & SSRI: $2 \pm 2$ & \\
Hamilton Depression Rating & Placebo: $2 \pm 1$ & $t_{1,17}=1.2, p=0.25$ \\
Scale & SSRI: $42 \pm 25$ & \\
Mood Spectrum Self-Report & Placebo: $37 \pm 22$ & \\
\end{tabular}

$\mathrm{SSRI}=$ selective serotonin reuptake inhibitor.

${ }^{*}$ Values are mean \pm standard deviation; $n=19$, within-subjects. 

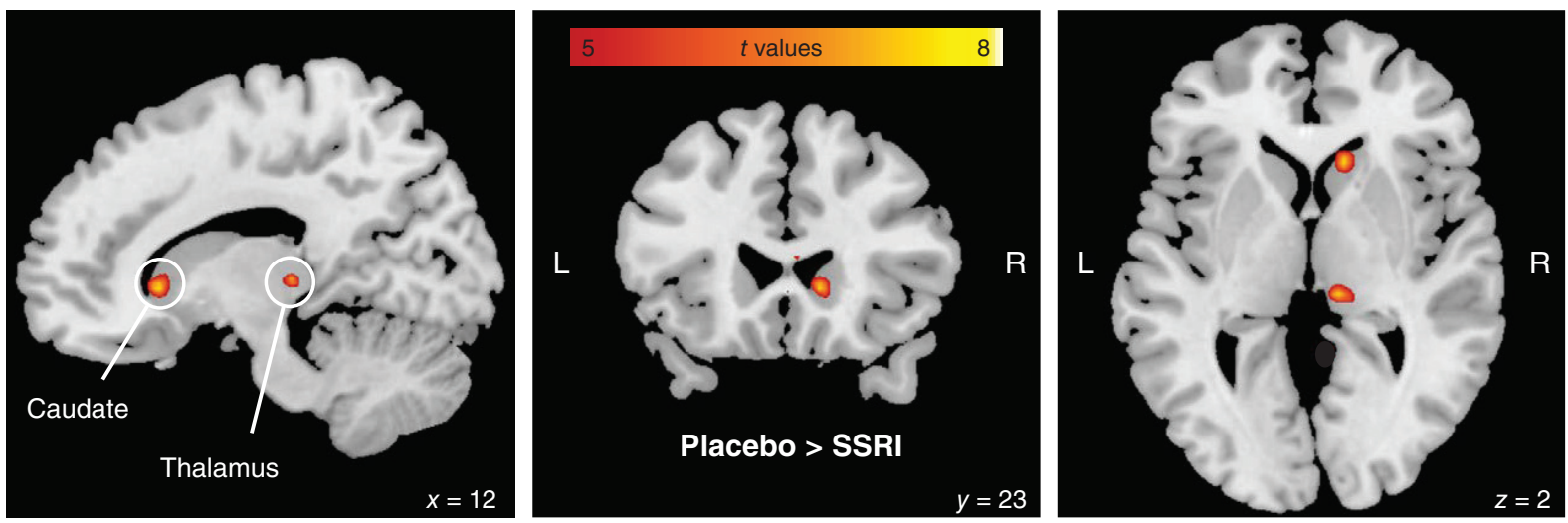

Fig. 2: Brain response to punishment feedback. Orthogonal brain sections showed reduced blood-oxygen-level-dependent signal difference between the monetary loss and neutral conditions after a single oral dose of escitalopram $(20 \mathrm{mg})$ compared with placebo. Significant effects were obtained in the right posterior thalamus $(x, y, z=10,-26,4)$ and the right caudate head $(x, y, z=12,22,0)$ using family-wise error correction at peak level with $p<0.05$. Cluster-defining threshold $p<0.001$ (uncorrected). SSRI = selective serotonin reuptake inhibitor.

$x, y, z=12,22,0)$ compared with placebo at the whole-brain level (Figure 2). We found no significant voxels that showed a BOLD increase with escitalopram, even without correction for multiple comparisons.

Investigating the win-neutral contrast revealed no significant medication effects, even without correction for multiple comparisons.

\section{Discussion}

In this placebo-controlled, crossover, double-blind pharmacofMRI study, we report the effects of an acute single dose of the SSRI escitalopram on neural response to reward and punishment feedback in healthy human participants. The main findings of this study were that acutely administered escitalopram reduced BOLD response in the right caudate nucleus and the right thalamus during monetary loss, and it did not induce changes in BOLD response to feedback on monetary wins. These results were consistent with previous work on the effects of SSRIs on the emotional processing of aversive stimuli, ${ }^{44}$ and they provide additional support for the neuropsychological theory that acute administration of SSRI reduces negativity bias.

Furthermore, our data extend this theory by suggesting a specific role for acute serotonin transporter occupancy in the modulation of healthy neural response to punishment feedback. Given that SSRIs are used as a first line of treatment for depression and anxiety disorders, a clinical response is typically expected after several weeks. ${ }^{45}$ This delayed onset of symptom relief in response to SSRI treatment is in contrast to the time it takes for the majority of SSRI target sites (the serotonin transporters) to be blocked to up to approximately $80 \%$. This percentage is within the occupancy range established to be required for a therapeutic effect ${ }^{46}$ and can already occur 3 hours after intake of the first clinical dose. ${ }^{5}$ The discrepancy in time of onset between the acute occupancy of the serotonin transporter and a clinical response has been explained by the time required for presynaptic serotonin $1 \mathrm{~A}$ autoreceptors to desensitize: it is postulated that the acute blockage of serotonin reuptake triggers a negative feedback mechanism via attenuated serotonergic firing, mediated by presynaptic serotonin $1 \mathrm{~A}$ autoreceptors. ${ }^{47}$ The resulting serotonin $1 \mathrm{~A}$ receptor-desensitization theory is supported by indirect evidence demonstrating decreases in the functional sensitivity of serotonin $1 \mathrm{~A}$ autoreceptors following chronic SSRI treatment in rodents. ${ }^{48}$ It is also supported by preliminary findings from positron emission tomography studies in people with MDD demonstrating a decrease in serotonin 1A receptor binding in the dorsal raphe nuclei (albeit unrelated to the antidepressant response) $)^{49}$ and decreased serotonin $1 \mathrm{~A}$ receptor binding limited to a significant effect in the hippocampus in people with anxiety disorders. ${ }^{50}$

However, at a cognitive psychological level it has been argued $^{51}$ that acute effects can be detected after a single SSRI dose. Pioneering work by Harmer and colleagues ${ }^{52,53}$ has shown that acute SSRI administration modulates implicit negative attention bias. Additional work in humans has shown that acute tryptophan depletion (i.e., acute decreases in serotonin levels) results in enhanced brain responses to threat-related stimuli, punishment predictive learning and interference from sad distractors (reviewed in Cools and colleagues ${ }^{54}$ ). Further evidence for this specific effect on negative bias is provided by a study in rats, ${ }^{55}$ which demonstrated that acute citalopram administration influenced negative feedback sensitivity, and subchronic administration influenced reward sensitivity. Moreover, the authors observed that dose level was an essential factor for sensitivity to negative feedback; a low single dose was associated with increased negative feedback sensitivity, and a high single dose was associated with decreased negative feedback sensitivity. ${ }^{55}$ This body of work, combined 
with our findings, suggests that acute SSRI administration may be specifically related to negative bias modulation, and that a single high SSRI dose (as used in our study) may already cause phasic increases in serotonergic signalling.

Our findings demonstrate additional support for this acute timeline and show that a single oral dose of escitalopram modulates the functional response to negative feedback in brain areas typically implicated in processing loss, a cognitive process highly relevant to implicit negative bias. In an exploratory fashion, we performed a loss $>$ reward follow-up analysis to the significant loss $>$ neutral findings, because this may provide further insight into whether escitalopraminduced dampening to loss is greater than to reward, compared with placebo. We did not find a significant result, although this could have been influenced by our sample size. As well, because we had observed a right lateralization in our original results, we re-ran this analysis using a less conservative statistical threshold. We observed a bilateral response in the thalamus and the caudate at $p<0.001$ (uncorrected), with the caudate clusters extending to the ventral striatum. However, given that this was not within our a priori criteria, these findings must be discussed with caution, and future studies with larger samples may be required for further clarification. Regardless, our current findings extend the observation that a single oral SSRI dose can affect emotional processing and may reduce implicit negative bias, ${ }^{52,53}$ showing that this acute intervention has a localized effect in the neural reward circuit, namely during punishment feedback.

Serotonergic neurotransmission has long been considered a crucial substrate for aversive processing and negative motivation, based on evidence from pharmacological depletion and challenge paradigms in humans. ${ }^{16,56,57} \mathrm{~A}$ blunted hedonic response to rewards, as well as enhanced sensitivity to punishment, describes a negative bias in reward processing that is common in depression. ${ }^{1}$ Recent models in computational psychiatry propose that negative mood reflects the cumulative effect of differences between reward outcomes and expectations. ${ }^{58,59}$ These models suggest a bidirectional interaction between mood and reward processing that may play an important adaptive role in healthy behaviour, and whose dysfunction might contribute to depressive disorders. ${ }^{60} \mathrm{How}-$ ever, the effect of SSRIs on the negative bias in processing rewards is less well understood than for processing emotions. By specifically investigating the modulation of the hemodynamic response to reward and punishment feedback, we found that an acute dose of SSRI attenuated the BOLD response to punishment feedback in the caudate head (which is part of the striatum) and the thalamus. This was in line with previous findings, which demonstrated blunted BOLD responses to both positive and negative feedback in the medial caudate $^{61}$ and the caudate and nucleus accumbens ${ }^{62}$ in (unmedicated) patients with MDD compared to healthy controls. A recent meta-analysis summarized these findings by reporting the caudate as the only significant region that differed between people with MDD and healthy controls during feedback processing, with decreased caudate activity in people with MDD. ${ }^{63}$ These findings are interpreted as evidence for reduced reinforcement of actions in people with MDD. More specifically, McCabe and colleagues ${ }^{64}$ found enhanced responses in the caudate and blunted responses in the lateral orbitofrontal cortex to primary aversive stimuli (e.g., mouldy strawberries) in patients with remitted MDD. The authors suggested that the caudate might play a role in automatic negative bias, and that blunted cortical responses represented the inability to integrate potential aversive information into appropriate actions. Although studies investigating unmedicated or remitted patients with MDD provide examples of how serotonergic neurotransmission may affect feedback processing in patient populations, we still lack a comprehensive basic understanding of how changes in acute serotonergic signalling affect reward and punishment feedback processing in healthy individuals.

Kumar and colleagues ${ }^{65}$ administered SSRIs to healthy participants for 3 consecutive days and found blunted responses related to reward prediction error in a similar network to that observed in medicated patients with MDD. In response to the SSRI, healthy participants showed a neural BOLD pattern that seemed to display an intermediate state between drug-naive healthy participants and patients with MDD taking SSRIs. This suggests that a "normal" processing of feedback might require a narrow window of serotonergic tone, and any imbalance would cause potential disruptions in feedback processing. Dayan and colleagues ${ }^{66}$ proposed that people with normal serotonin levels should reflexively inhibit ("prune") choices with poor expected outcomes. Any rapid drop in serotonin levels would compromise this adaptive mechanism of underexploring negative environments and would lead instead to the subjective experience of more negative events. Our results support this hypothesis by demonstrating that acute serotonergic manipulation alters the neural response to negative feedback. These data complement a recent study reporting that a single dose of escitalopram increased lose-shift behaviour after negative feedback, while win-shift behaviour remained unchanged in healthy participants. ${ }^{67}$ At a more general level, our results were in accordance with the role of serotonin in aversive processing, more specifically in inhibiting behaviours associated with adverse consequences ${ }^{13,14}$ and possibly linked to promoting patience. ${ }^{68}$

\section{Limitations}

One limitation of the study was that the BOLD signal is a nonquantitative measure that integrates both blood volume and oxygen extraction, and it does not allow distinction between the 2 measures. We acknowledge that part of the signal we observed may have been due to SSRI effects on global cerebral blood flow (e.g., via a change in blood-vessel tone) ${ }^{69}$ However, it is unlikely that the signal change we observed during punishment feedback was entirely driven by a global change in cerebral blood flow, given the previous work of our group and others demonstrating differentiated regional effects ${ }^{70,71}$ and no evidence that the same pharmacological challenge altered resting-state measures, such as the amplitude of low-frequency fluctuations, ${ }^{70}$ which would have been highly sensitive to such effects. Finally, even if part of the signal were driven by underlying early changes in 
neurovascular coupling in the thalamus and caudate, this was still a finding worth describing and reporting, because it may still be relevant for understanding the initial brain response to the first dose of escitalopram.

Second, we chose to study healthy young participants, and acknowledge that any interpretation of our results is limited to a healthy population and may not apply to middle-aged or older populations, or to patients. Several groups have demonstrated that acute or short-term treatment reduces negative biases in information processing, paralleled by changes of the brain response in the amygdala, thalamus, cingulate and insula in healthy participants. ${ }^{22,72,73}$ As well, similar acute BOLD response patterns in the amygdala, thalamus, cingulate and insula during affective processing were predictive of a clinical response to escitalopram after 6 weeks of treatment in patients with MDD. Still, the effects of SSRIs on serotonergic neurotransmission might differ between healthy individuals and clinical populations. ${ }^{65}$

Third, we chose a task that allowed assessment of the brain response but did not include a behavioural assessment or measures of potential arousing or sedating effects of SSRI intake. Consequently, our results are limited to the interpretation of the healthy brain response to punishment feedback during maximum SSRI levels in the periphery and a $75 \%$ to $80 \%$ block of the central serotonin transporter state. Changes at a neural level can occur without concurrent behavioural change, specifically when assessed on an acute timescale. Given the multilayered concepts of reward and punishment learning, the wide variety of relatively complex paradigms currently applied to tease those layers apart, and the subsequent heterogeneous findings, ${ }^{74}$ we opted for a well-established, simple and straightforward task. ${ }^{38,39}$ The task, combined with a longitudinal within-subject design, allowed for a robust assessment of the acute effects of a single dose of escitalopram during punishment feedback in a neural circuit relevant to punishment processing and at a considerable effect size and a relatively conservative statistical threshold at the whole-brain level. However, we acknowledge that the thalamus finding did not survive FWE correction at the cluster level. We chose to focus on FWE correction at the voxel level, but as previous work $^{43}$ has shown, cluster inference is prone to producing false-positive results, and the voxel inference shows FWE rates in the expected order of magnitude.

Finally, we acknowledge that the activity of SSRIs on the regulation of neural excitation and inhibition is multifactorial and complex, and that serotonin transporter blockade can affect several neurotransmitter systems beyond serotonin, such as dopamine and noradrenaline. Future studies implementing multiple and specific tracer applications for quantitative neuroimaging tools (such as positron emission tomography or magnetic resonance spectroscopy) are needed to shed light on the complex interplay of synergistic neurotransmitters in different aspects of reward and punishment interaction.

\section{Conclusion}

Our study provides an important and novel contribution to the understanding of how acute SSRI administration affects the human brain, and specifically the reward system, by demonstrating that a single dose of escitalopram alters the brain response to punishment but not reward feedback in healthy individuals. Our results complement recent theories of antidepressant action by showing an acute blunting effect of SSRI administration on negative feedback processing and demonstrate a role for a single dose of escitalopram in affecting the neural response to punishment. These findings are in accordance with the role of serotonin in aversive processing, and more specifically provide support for the hypothesis of the protective action of serotonin for the healthy brain in the face of negative events. Furthermore, they provide a crucial next step toward testing clinical translation for such paradigms of punishment feedback, particularly in patients with increased punishment sensitivity, such as those with obsessivecompulsive disorder, ${ }^{75}$ eating disorders ${ }^{76}$ or depression. ${ }^{1}$

Affiliations: From the Emotion Neuroimaging Lab, Max Planck Institute for Human Cognitive and Brain Sciences, Leipzig, Germany (Lewis, Zsido, Sacher); the International Max Planck Research School on Neuroscience of Communication: Function, Structure, and Plasticity, Leipzig, Germany (Lewis, Zsido); the Department of Psychiatry and Psychotherapy, Medical School, University of Tuebingen, Germany (Lewis); the Max Planck Institute for Human Cognitive and Brain Sciences, Leipzig, Germany (Mueller, Reinelt, Villringer); the Max Planck School of Cognition, Leipzig, Germany (Zsido); the Division of Clinical Pharmacology, Rudolf-Boehm-Institute of Pharmacology and Toxicology, University of Leipzig, Leipzig, Germany (Regenthal); the Department of Psychology, School of Psychological Sciences, University of Haifa, Haifa, Israel (Okon-Singer); the Integrated Brain and Behavior Research Center (IBBR), University of Haifa, Haifa, Israel (Okon-Singer); the Department of Psychiatry, University of Pittsburgh School of Medicine, Pittsburgh, PA, USA (Forbes); and the Clinic for Cognitive Neurology, University of Leipzig, Leipzig, Germany (Villringer, Sacher).

Funding: C. Lewis and J. Sacher were supported by the Branco Weiss Fellowship-Society in Science, National Association for Research on Schizophrenia and Depression (NARSAD) Young Investigator Grant 25032 from the Brain and Behavior Research Foundation, and by a Minerva Research Group grant from the Max Planck Society (all awarded to J. Sacher).

Competing interests: E. Forbes declares an honorarium for editorial activities for the Association for Psychological Science; paid consultancy for DSMB, Durham VA (sponsor), Otsuka (funder); an honorarium for mentoring activities as part of Research Centre, Brown University; research funding from the National Institutes of Health; and an honorarium for a grant review from the National Institutes of Health, all outside the published work. No other competing interests declared.

Contributors: E. Forbes, A. Villringer and J. Sacher designed the study. R. Regenthal and J. Sacher acquired the data, which C. Lewis, K. Mueller, R. Zsido, J. Reinelt, H. Okon-Singer and J. Sacher analyzed. C. Lewis, R. Zsido, R. Regenthal and J. Sacher wrote the article, which all authors reviewed. All authors approved the final version to be published and can certify that no other individuals not listed as authors have made substantial contributions to the paper.

Content licence: This is an Open Access article distributed in accordance with the terms of the Creative Commons Attribution (CC BY-NC-ND 4.0) licence, which permits use, distribution and reproduction in any medium, provided that the original publication is properly cited, the use is noncommercial (i.e., research or educational use), and no modifications or adaptations are made. See: https:/ / creativecommons.org/licenses/by-nc-nd/4.0/ 


\section{References}

1. Eshel N, Roiser JP. Reward and punishment processing in depression. Biol Psychiatry 2010;68:118-24.

2. Dillon DG, Rosso IM, Pechtel P, et al. Peril and pleasure: an rdocinspired examination of threat responses and reward processing in anxiety and depression. Depress Anxiety 2014;31:233-49.

3. Kranz GS, Kasper S, Lanzenberger R. Reward and the serotonergic system. Neuroscience 2010;166:1023-35.

4. Nutt DJ. The neuropharmacology of serotonin and noradrenaline in depression. Int Clin Psychopharmacol 2002;17(Suppl 1):S1-12.

5. Klein N, Sacher J, Geiss-Granadia T, et al. In vivo imaging of serotonin transporter occupancy by means of SPECT and [123I]ADAM in healthy subjects administered different doses of escitalopram or citalopram. Psychopharmacology (Berl) 2006;188:263-72.

6. El Mansari M, Sánchez C, Chouvet G, et al. Effects of acute and long-term administration of escitalopram and citalopram on serotonin neurotransmission: an in vivo electrophysiological study in rat brain. Neuropsychopharmacology 2005;30:1269-77.

7. Hajós M, Gartside SE, Sharp T. Inhibition of median and dorsal raphe neurones following administration of the selective serotonin reuptake inhibitor paroxetine. Naunyn Schmiedebergs Arch Pharmacol 1995;351:624-9.

8. Beyer CE, Cremers TI. Do selective serotonin reuptake inhibitors acutely increase frontal cortex levels of serotonin? Eur J Pharmacol 2008;580:350-4.

9. Invernizzi R, Belli S, Samanin R. Citalopram's ability to increase the extracellular concentrations of serotonin in the dorsal raphe prevents the drug's effect in the frontal cortex. Brain Res 1992; 584:322-4.

10. Marek GJ, Martin-Ruiz R, Abo A, et al. The selective 5-HT 2A receptor antagonist M100907 enhances antidepressant-like behavioral effects of the SSRI fluoxetine. Neuropsychopharmacology 2005;30:2205-15.

11. Garcia-Garcia AL, Newman-Tancredi A, Leonardo ED. P5-HT 1A receptors in mood and anxiety: recent insights into autoreceptor versus heteroreceptor function. Psychopharmacology (Berl) 2014; 231:623-36.

12. Koch S, Perry KW, Nelson DL, et al. R-fluoxetine increases extracellular DA, NE, as well as 5-HT in rat prefrontal cortex and hypothalamus: an in vivo microdialysis and receptor binding study. Neuropsychopharmacology 2002;27:949-59.

13. Daw ND, Kakade S, Dayan P. Opponent interactions between serotonin and dopamine. Neural Netw 2002;15:603-16.

14. Deakin JF, Graeff FG. 5-HT and mechanisms of defence. J Psychopharmacol 1991;5:305-15.

15. Cools R, Robinson OJ, Sahakian B. Acute tryptophan depletion in healthy volunteers enhances punishment prediction but does not affect reward prediction. Neuropsychopharmacology 2008;33:2291-9.

16. Crockett MJ, Clark L, Robbins TW. Reconciling the role of serotonin in behavioral inhibition and aversion: acute tryptophan depletion abolishes punishment-induced inhibition in humans. J Neurosci 2009;29:11993-9.

17. Moran RJ, Kishida KT, Lohrenz T, et al. The protective action encoding of serotonin transients in the human brain. Neuropsychopharmacology 2018;43:1425-35.

18. Soubrié P. Reconciling the role of central serotonin neurons in human and animal behavior. Behav Brain Sci 1986;9:319-35.

19. Cools R, Nakamura K, Daw ND. Serotonin and dopamine: unifying affective, activational, and decision functions. Neuropsychopharmacology 2011;36:98-113.

20. Nakamura K. The role of the dorsal raphe nucleus in reward-seeking behavior. Front Integr Nuerosci 2013;27:60.

21. Browne CJ, Fletcher PJ. Decreased incentive motivation following knockout or acute blockade of the serotonin transporter: role of the 5-HT2C receptor. Neuropsychopharmacology 2016;41:2566-76.

22. McCabe C, Mishor Z, Cowen PJ, et al. Diminished neural processing of aversive and rewarding stimuli during selective serotonin reuptake inhibitor treatment. Biol Psychiatry 2010;67:439-45.

23. Liu Z, Zhou J, Li Y, et al. Dorsal raphe neurons signal reward through 5-HT and glutamate. Neuron 2014;81:1360-74.

24. Zhou J, Jia C, Feng Q, et al. Prospective coding of dorsal raphe reward signals by the orbitofrontal cortex. J Neurosci 2015;35:2717-30.

25. Browne CJ, Abela AR, Chu D, et al. Dorsal raphe serotonin neurons inhibit operant responding for reward via inputs to the ventral tegmental area but not the nucleus accumbens: evidence from studies combining optogenetic stimulation and serotonin reuptake inhibition. Neuropsychopharmacology 2019;44:793-804.

26. Evers EA, Cools R, Clark L, et al. Serotonergic modulation of prefrontal cortex during negative feedback in probabilistic reversal learning. Neuropsychopharmacology 2005;30:1138-47.

27. Macoveanu J, Rowe JB, Hornboll B, et al. Playing it safe but losing anyway-serotonergic signaling of negative outcomes in dorsomedial prefrontal cortex in the context of risk-aversion. Eur Neuropsychopharmacol 2013;23:919-30.

28. Del-Ben CM, Deakin JF, McKie S, et al. The effect of citalopram pretreatment on neuronal responses to neuropsychological tasks in normal volunteers: an FMRI study. Neuropsychopharmacology 2005;30:1724-34.

29. Marutani T, Yahata N, Ikeda Y, et al. Functional magnetic resonance imaging study on the effects of acute single administration of paroxetine on motivation-related brain activity. Psychiatry Clin Neurosci 2011;65:191-8.

30. Kasper S, Sacher J, Klein N, et al. Differences in the dynamics of serotonin reuptake transporter occupancy may explain superior clinical efficacy of escitalopram versus citalopram. Int Clin Psychopharmacol 2009;24:119-25.

31. Forbes EE, Olino TM, Ryan ND, et al. Reward-related brain function as a predictor of treatment response in adolescents with major depressive disorder. Cogn Affect Behav Neurosci 2010; 10:107-18.

32. Knutson B, Westdorp A, Kaiser E, et al. FMRI visualization of brain activity during a monetary incentive delay task. Neuroimage 2000;12:20-7.

33. Aronson S, Delgado P. Escitalopram. Drugs Today (Barc) 2004;40: 121-31.

34. Wittchen HU. Strukturiertes klinisches Interview für DSM-IV: SKID. Achse I: Psychische Störungen: Interviewheft und Beurteilungsheft; eine deutschsprachige, erweiterte Bearbeitung der amerikanischen Originalversion des SCID-I. Hogrefe: Verlag für Psychologie; 1997.

35. Hamilton M. A rating scale for depression. J Neurol Neurosurg Psychiatry 1960;23:56-62.

36. Dell'Osso L, Armani A, Rucci P, et al. Measuring mood spectrum: comparison of interview (SCI-MOODS) and self-report (MOODS-SR) instruments. Compr Psychiatry 2002;43:69-73.

37. Spielberger CD, Gorsuch RL, Lushene RD, et al. Manual for the state trait anxiety inventory (STAI). Palo Alto (CA): Consulting Psychologists Press; 1983.

38. Forbes EE, Hariri AR, Martin SL, et al. Altered striatal activation predicting real-world positive affect in adolescent major depressive disorder. Am J Psychiatry 2009;166:64-73.

39. Forbes EE, Rodriguez EE, Musselman S, et al. Prefrontal response and frontostriatal functional connectivity to monetary reward in abstinent alcohol-dependent young adults. PLoS One 2014;9: e94640.

40. Henson R. Efficient experimental design for fMRI. In: Penny WD, Friston KJ, Ashburner J, et al, editors. Statistical parametric mapping: the analysis of functional brain images. Amsterdam: Elsevier; 2007.

41. Power JD, Barnes KA, Snyder AZ, et al. Spurious but systematic correlations in functional connectivity MRI networks arise from subject motion. Neuroimage 2012;59:2142-54.

42. Ashburner J, Friston KJ. Unified segmentation. Neuroimage 2005;26:839-51.

43. Eklund A, Nichols TE, Knutsson H. Cluster failure: why fMRI inferences for spatial extent have inflated false-positive rates. Proc Natl Acad Sci U S A 2016;113:7900-5.

44. Harmer CJ, O'Sullivan U, Favaron E, et al. Effect of acute antidepressant administration on negative affective bias in depressed patients. Am J Psychiatry 2009;166:1178-84.

45. Nestler EJ. Antidepressant treatments in the 21st century. Biol Psychiatry 1998;44:526-33.

46. Meyer JH, Wilson AA, Sagrati S, et al. Serotonin transporter occupancy of five selective serotonin reuptake inhibitors at different doses: an [11C]DASB positron emission tomography study. Am J Psychiatry 2004;161:826-35.

47. Blier P, de Montigny C, Chaput $\mathrm{Y}$. Modifications of the serotonin system by antidepressant treatments: implications for the therapeutic response in major depression. J Clin Psychopharmacol 1987; 7(Suppl):24S-35S.

48. Artigas F, Romero L, de Montigny C, et al. Acceleration of the effect of selected antidepressant drugs in major depression by 5-HT1A antagonists. Trends Neurosci 1996;19:378-83. 
49. Gray NA, Milak MS, DeLorenzo C, et al. Antidepressant treatment reduces serotonin-1A autoreceptor binding in major depressive disorder. Biol Psychiatry 2013;74:26-31.

50. Spindelegger C, Lanzenberger R, Wadsak W, et al. Influence of escitalopram treatment on 5-HT1A receptor binding in limbic regions in patients with anxiety disorders. Mol Psychiatry 2009; 14:1040-50.

51. Harmer CJ, Goodwin GM, Cowen PJ. Why do antidepressants take so long to work? A cognitive neuropsychological model of antidepressant drug action. Br J Psychiatry 2009;195:102-8.

52. Harmer CJ, Bhagwagar Z, Perrett D, et al. Acute SSRI administration affects the processing of social cues in healthy volunteers. Neuropsychopharmacology 2003;28:148-52.

53. Harmer CJ, Shelley NC, Cowen PJ, et al. Increased positive versus negative affective perception and memory in healthy volunteers following selective serotonin and norepinephrine reuptake inhibition. Am J Psychiatry 2004;161:1256-63.

54. Cools R, Roberts AC, Robbins TW. Serotoninergic regulation of emotional and behavioural control processes. Trends Cogn Sci 2008;12:31-40.

55. Bari A, Theobald DE, Caprioli D, et al. Serotonin modulates sensitivity to reward and negative feedback in a probabilistic reversal learning task in rats. Neuropsychopharmacology 2010;35:1290-301.

56. Chamberlain SR, Muller U, Blackwell AD, et al. Neurochemical modulation of response inhibition and probabilistic learning in humans. Science 2006;311:861-3.

57. Macoveanu J, Rowe JB, Hornboll B, et al. Playing it safe but losing anyway-serotonergic signaling of negative outcomes in dorsomedial prefrontal cortex in the context of risk-aversion. Eur Neuropsychopharmacol 2013;23:919-30.

58. Huys QJ, Daw ND, Dayan P. Depression: a decision-theoretic analysis. Annu Rev Neurosci 2015;38:1-23.

59. Montague PR, Dolan RJ, Friston KJ, et al. Computational psychiatry. Trends Cogn Sci 2012;16:72-80.

60. Eldar E, Rutledge RB, Dolan RJ, et al. Mood as representation of momentum. Trends Cogn Sci 2016;20:15-24.

61. Elliott R, Sahakian BJ, Michael A, et al. Abnormal neural response to feedback on planning and guessing tasks in patients with unipolar depression. Psychol Med 1998;28:559-71.

62. Pizzagalli DA, Holmes AJ, Dillon DG, et al. Reduced caudate and nucleus accumbens response to rewards in unmedicated individuals with major depressive disorder. Am J Psychiatry 2009;166:702-10.
63. Keren H, O'Callaghan G, Vidal-Ribas $\mathrm{P}$, al. Reward processing in depression: a conceptual and meta-analytic review across fMRI and EEG studies. Am J Psychiatry 2018:175:1111-20.

64. McCabe C, Cowen PJ, Harmer CJ. Neural representation of reward in recovered depressed patients. Psychopharmacology (Berl) 2009; 205:667-77.

65. Kumar P, Waiter G, Ahearn T, et al. Abnormal temporal difference reward-learning signals in major depression. Brain 2008; 131:2084-93.

66. Dayan P, Huys QJ. Serotonin, inhibition, and negative mood. PLOS Comput Biol 2008;4:e4.

67. Skandali N, Rowe JB, Voon V, et al. Dissociable effects of acute SSRI (escitalopram) on executive, learning and emotional functions in healthy humans. Neuropsychopharmacology 2018;43:2645-51.

68. Miyazaki KW, Miyazaki K, Tanaka KF, et al. Optogenetic activation of dorsal raphe serotonin neurons enhances patience for future rewards. Curr Biol 2014;24:2033-40.

69. Blanco VM, Stern JE, Filosa JA. Tone-dependent vascular responses to astrocyte-derived signals. Am J Physiol Heart Circ Physiol 2008;294:H2855-63.

70. Schaefer A, Burmann I, Regenthal R, et al. Serotonergic modulation of intrinsic functional connectivity. Curr Biol 2014;24:2314-8.

71. Windischberger C, Lanzenberger R, Holik A, et al. Area-specific modulation of neural activation comparing escitalopram and citalopram revealed by pharmaco-fMRI: a randomized cross-over study. Neuroimage 2010;49:1161-70.

72. Godlewska BR, Browning M, Norbury R, et al. Early changes in emotional processing as a marker of clinical response to SSRI treatment in depression. Transl Psychiatry 2016;6:e957.

73. Harmer CJ, Cowen PJ. 'It's the way that you look at it' - a cognitive neuropsychological account of SSRI action in depression. Philos Trans R Soc Lond B Biol Sci 2013;368:20120407.

74. Kubanek J, Snyder LH, Abrams RA. Reward and punishment act as distinct factors in guiding behavior. Cognition 2015;139: 154-67.

75. Fullana MA, Mataix-Cols D, Caseras X, et al. High sensitivity to punishment and low impulsivity in obsessive-compulsive patients with hoarding symptoms. Psychiatry Res 2004;129:21-7.

76. Monteleone AM, Cardi V, Volpe U, et al. Attachment and motivational systems: relevance of sensitivity to punishment for eating disorder psychopathology. Psychiatry Res 2018;260:353-9. 